\title{
Increasing Detection and Confirmation of Tuberculosis in Children in Southern Ethiopia: Pooled Samples Tested Using Microscopy and Xpert
}

\author{
Daniel G. Datiko1*, Mulualem A. Gadena1, Mubarek A. Yassin'1, Kefyalew T. Garie², \\ Moges D. Ormago², Luis E. Cuevas ${ }^{3}$ \\ ${ }^{1}$ REACH Ethiopia, Hawassa, Ethiopia \\ ${ }^{2}$ University of Hawassa, Hawassa, Ethiopia \\ ${ }^{3}$ Liverpool School of Tropical Medicine, Pembroke Place, Liverpool, UK \\ Email: *danieljohn42@yahoo.com
}

How to cite this paper: Datiko, D.G., Gadena, M.A., Yassin, M.A., Garie, K.T., Ormago, M.D. and Cuevas, L.E. (2018) Increasing Detection and Confirmation of Tuberculosis in Children in Southern Ethiopia: Pooled Samples Tested Using Microscopy and Xpert. Journal of Tuberculosis Research, 6, 215-226.

https://doi.org/10.4236/jtr.2018.63020

Received: July 26, 2018

Accepted: September 18, 2018

Published: September 21, 2018

Copyright $\odot 2018$ by authors and Scientific Research Publishing Inc. This work is licensed under the Creative Commons Attribution International License (CC BY 4.0).

http://creativecommons.org/licenses/by/4.0/

\section{(c) (i) Open Access}

\begin{abstract}
Background: Childhood tuberculosis accounts for about 10\% of estimated TB cases in the world. Despite advances in diagnostics, childhood TB remains a challenge. We evaluated pooling method and testing with GeneXpert MTB/RIF in southern Ethiopia. Methods: This is a cross-sectional study in presumptive TB children $<15$ years. Structured questionnaire was used to collect socio-demographic and clinical data. Two sputum or gastric aspirate sample were collected and examined by ZN and Xpert MTB/RIF for $1^{\text {st }}, 2^{\text {nd }}$ and pooled samples. Results: Of 340 presumptive TB cases enrolled, 96 and 244 children submitted gastric aspirate and sputum samples respectively. Of 1020 samples collected (282 gastric aspirate and 738 sputum samples), 38 (3.7\%) were positive by Xpert (10 (3.5\%) from gastric aspirate and 28 (3.8\%) from sputum sample). Similarly, 8 (1.2\%) of sputum samples were positive by ZN but none from gastric aspirate. Of 244 children who submitted sputum samples, $3(1.2 \%)$ were bacteriologically positive compared to $12(4.9 \%)$ by Xpert. Of 96 children who submitted gastric aspirate samples, none were positive by ZN while 5 (5.2\%) were positive by Xpert. Of bacteriologically confirmed TB cases $0.9 \%$ was by $\mathrm{ZN}$ and $4.7 \%$ by Xpert, an increase of $3.8 \%$. Pooled testing increased positivity by $0.3 \%$ for $\mathrm{ZN}$ and $1.5 \%$ by Xpert compared to the $1^{\text {st }}$ sample. Conclusions: Xpert MTB/RIF testing increases yield compared to $\mathrm{ZN}$ testing for gastric aspirate samples. The same-day approach and pooling samples improves efficient use of cartridge, reduce the number of visits for seeking diagnosis and save resources.
\end{abstract}




\section{Keywords}

TB, Children, GeneXpert MTB/RIF, Pooled Sample

\section{Background}

Childhood tuberculosis (TB) accounts for about $10 \%$ of globally estimated new $\mathrm{TB}$ cases [1] and remains among top diseases causing the highest morbidity and mortality among children admitted for treatment [2]. However, childhood TB prevention and control did not get adequate attention. The main reasons are lack of the capacity to generate quality epidemiologic data mainly due to the inherent difficulties in diagnosing pediatric TB, limited health workers and lack of the facilities or diagnostic tools [3] [4].

Decades after the implementation of decentralized TB prevention and control efforts, National TB Programmes are still far behind reaching goals set for reaching missed $\mathrm{TB}$ cases in the communities. As a result, childhood TB prevention and control were not a priority as the capacity to pick adult TB is yet to be improved. This creates safe haven for TB transmission from adults to children [5]. In addition, the capacity of children to produce adequate sputum samples and low bacilli load in the specimens complicated the case finding in children. Thus, childhood TB cases are inadequately detected and under reported by $\mathrm{Na}$ tional TB programmes [2] [6].

Using the present diagnostic tools over 95\% treatment success rates of childhood tuberculosis with PTB and EPTB is traceable in developed countries. In developing countries, rates of successful treatment are often much lower [7] and childhood TB diagnosis is not well prioritized and implemented [8] [9].

TB is one of the leading causes of morbidity and mortality in children. However, only about $5 \%$ of the estimated cases are detected due to the problems related to the health system and the community. Therefore, alternative diagnostic approaches are required [7] [10]. 31\% of globally estimated childhood TB is found in Africa, resource constrained high burden continent [2].

Ethiopia like other developing countries, childhood tuberculosis was not given priority and the health facilities were not adequately organized to give access to the majority of rural communities. TB diagnosis relied on sputum smear microscopy, children often fail to produce sputum and smear microscopy has a low sensitivity in specimens, such as gastric aspirate, which is the method recommended for collection of specimens in children [11].

Recently Ethiopia stipulated Xpert mycobacterium/Rifampicin (MTB/RIF) as the initial diagnostic test for children presumed to have $\mathrm{TB}$, in order to increase bacteriological confirmed pediatric TB cases. Unfortunately, it is well known that testing only one specimen with Xpert in children results in a small proportion (typically about $20 \%$ ) of patients with a positive result and better ways to identify more children are needed [7]. Study in Nigeria showed that $115(16 \%)$ 
of 738 individual and 81 (44\%) of 185 pooled samples were Xpert positive for $M$. tuberculosis [12]. Cognizant of the low case finding and poor diagnostic yield, we aimed to evaluate if using pooled sample testing could increase the yield compared to individual tests and to evaluate Xpert results against the routine microscopy.

\section{Methods}

\subsection{Study Area}

This study was conducted in Sidama zone in southern Ethiopia. It has a population of about 3.5 million living in 19 rural districts and three town administrations. There are a total of 130 Health Centers and 5 district hospitals. Of these, 63 health facilities provide AFB diagnostic service; three have GeneXpert service and four have radiology service.

\subsection{Study Participants}

This is a cross-sectional study that enrolled children less than 15 years who had symptoms and signs of tuberculosis (cough of at least two weeks with or without other signs and symptoms). The children were recruited at health centers or from the community during house-to-house visit for contact investigation. Children who were diagnosed for $\mathrm{TB}$ or on treatment were excluded from the study. The sample size was estimated based on known Xpert positivity of $28 \%$ [13]. During the study period, 350 children from 0 to 14 years old symptomatic children were included in the study.

\subsection{Inclusion and Exclusion Criteria}

Children with cough of two weeks were included in the study. Children who do not have symptoms, seriously ill and parents did not give consent to participate in the study were excluded.

\subsection{Data Collection: Sample Collection and Processing}

Awareness creation sessions were conducted for health workers and community health workers in the area about how to identify pediatric presumptive TB case and link to nearby health facilities. Training was given to nurses and laboratory professions about the study. Pretested semi-structured questionnaire was used for data collection and sample collection, transport and testing.

Parents or guardians were informed about the sample collection process and its significance. Two spot-spot sputum sample or gastric expectorate (nasogastric tube was kept in place) were collected for each child in one hour difference.

The sample collection was done by experienced and trained nurses and laboratory technicians from Regional Institute of Public Health and health workers. In addition, the gastric aspirate sample were neutralized with PBS (phosphate buffer saline) ( $\mathrm{pH}=7.0)$ to ensure sample adequacy.

In this study the sample collection was conducted in the same day of recruit- 
ing the children in health centers and hospitals. However, the routine programme did not collect in health centers. The sample collection in hospitals requires visit for examination and waiting for admission, sample collection at least for two days plus days for testing and treatment initiation. Children were not followed up after completing diagnostic work up as per the research protocol.

Pooled samples were prepared from either expectorate sputum samples or gastric aspirates of each child. It was done by transferring half volume of the first sample and another half volume of the second sample into a separate sterile falcon tubes by using sterile disposable pipettes. All samples (two spot and one pooled) were examined by ZN stain and Gene Xpert (Figure 1).

\subsection{Laboratory Diagnosis of Bacteriologically Confirmed Cases}

Z-N stain [14] and GeneXpert MTB/RIF [15] was done as per the standard of procedure [16].

\subsection{Statistical Analysis}

Data entry and analysis was done using computer with SPSS version 20 software. Kappa values were done for evaluation of agreement between Testing of Pooled sample and individual sample using Smear microscopy and Xpert.

\subsection{Ethical Consideration}

This project was approved by SNNPR Institutional Review Board and official permission from the study sites was obtained. Informed consent was obtained

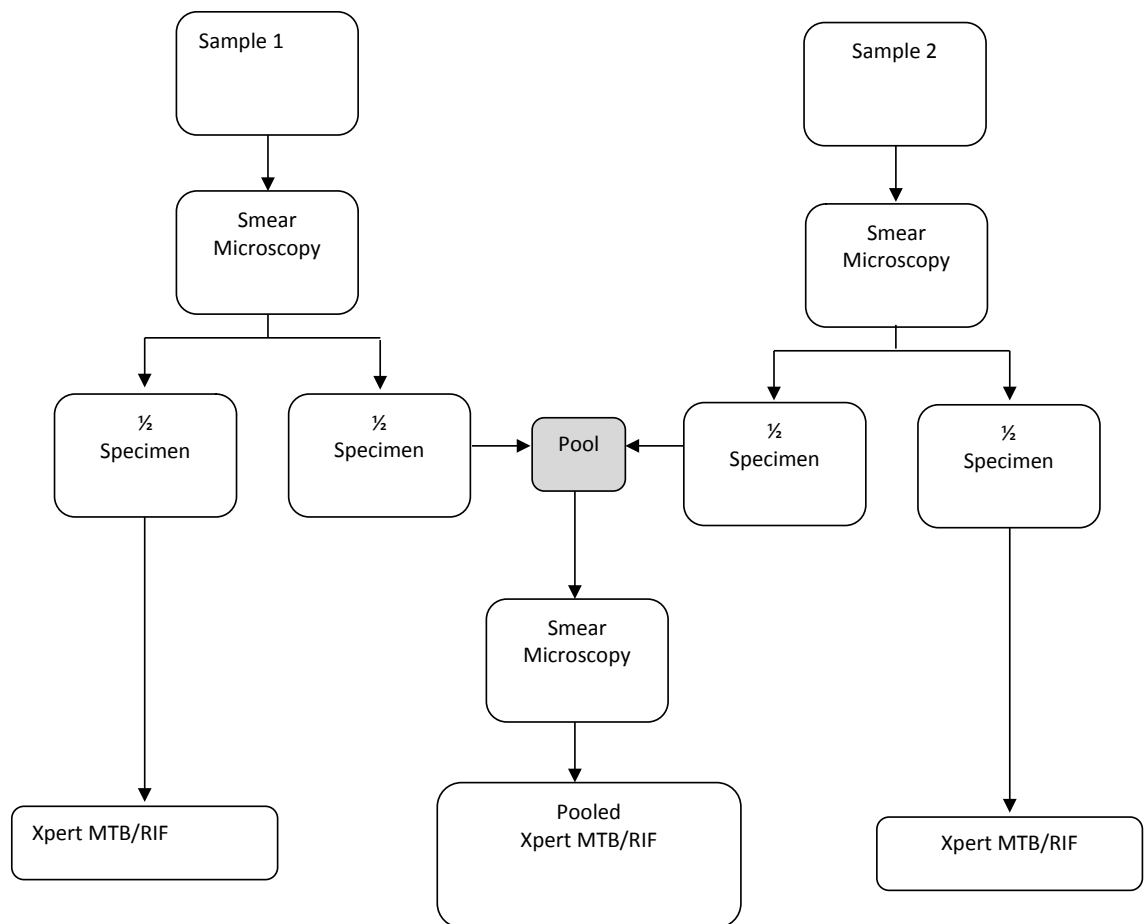

Figure 1. Flow chart showing the preparation and testing of the samples obtained from children in southern Ethiopia. 
from parent/guardian of study participants. Children who were diagnosed to have active TB were started on treatment as per the National TB guideline of Ethiopia.

\section{Result}

\subsection{Socio-Demographic Characteristics}

Of the 340 children enrolled, 173 (50.9\%) were females and 167 (49.1\%) were males. Of the total participants, 76 (22.4\%), $149(43.8 \%)$ and 115 (33.8\%) were in the age group of less or equal to four, five to nine and 10 to 14 years respectively. The mean and standard deviation of the participant's age were $7.7 \pm 3.7$ ranging from 2 months to 14 years.

From the total 264 study participants that were in 5 - 14 age group, $90(34 \%)$ were on school and 174 (66\%) were not on school. 201 (59.1\%) and 94 (27.6\%) were living with one and two or more room respectively. There were 148 (43.5\%) and $140(41.2 \%)$ participants with five or less and six and more household members were living together respectively (Table 1 ).

\subsection{TB Symptom Characteristics}

The most frequent clinical symptoms at presentation were cough of two or more weeks duration 340 (100\%), unexplained fever 296 (87.1\%), anorexia/appetite loss 298 (87.6\%), weight loss 291 (85.6\%) and Chest pain/difficult breathing 148 (43.5\%) and bloody sputum 28 (8.2\%). From the total 340 children that had cough, 220 (64.7\%), 87 (25.6\%) and 33 (9.7\%) had two to four, five to eight and greater than nine weeks duration of cough respectively (Table 2).

Out of the total participants, 147 (43.2\%) had contact history at the household. 139 (40.9\%) children reported that they had visited health facilities for

Table 1. Socio-demographic characteristics of presumptive TB children in southern Ethiopia.

\begin{tabular}{cccc}
\hline Category & Classification & Frequency & Percent \\
\hline Age & $0-4$ years & 76 & 22.4 \\
Sex & $5-9$ years & 149 & 43.8 \\
& $10-14$ years & 115 & 33.8 \\
Educational status & Male & 167 & 49.1 \\
& Female & 173 & 50.9 \\
Parents Occupation & No schooling & 91 & 26.8 \\
& Farmer & 249 & 73.2 \\
Parent's Married status & All other status & 224 & 65.9 \\
& Married & 116 & 34.1 \\
& All other status & 307 & 90.3 \\
House-hold members & $\leq 5$ & 33 & 9.3 \\
& $\geq 6$ & 148 & 43.5 \\
& & 192 & 56.5 \\
\hline
\end{tabular}


Table 2. TB symptoms and examinations done for children in southern Ethiopia.

\begin{tabular}{|c|c|c|c|}
\hline Variables & Classification & Frequency & Percent \\
\hline \multirow{2}{*}{ Cough } & Yes & 340 & 100 \\
\hline & No & 0 & 0 \\
\hline \multirow{2}{*}{ Bloody sputum } & Yes & 28 & 8.2 \\
\hline & No & 312 & 91.8 \\
\hline \multirow{2}{*}{ Fever } & Yes & 296 & 87.1 \\
\hline & No & 44 & 12.9 \\
\hline \multirow{2}{*}{ Loss of appetite Anorexia/appetite loss } & Yes & 298 & 87.6 \\
\hline & No & 42 & 12.4 \\
\hline \multirow{2}{*}{ Weight loss } & Yes & 291 & 85.6 \\
\hline & No & 49 & 14.4 \\
\hline \multirow[b]{2}{*}{ Chest pain } & Yes & 148 & 43.5 \\
\hline & No & 192 & 56.5 \\
\hline \multirow{2}{*}{ HF visited } & Yes & 139 & 40.9 \\
\hline & No & 201 & 59.1 \\
\hline \multirow{2}{*}{ TB treatment history } & Yes & 0 & 0 \\
\hline & No & 340 & 100 \\
\hline \multirow{2}{*}{ Contact history } & Yes & 147 & 43.2 \\
\hline & No & 193 & 56.8 \\
\hline \multirow{2}{*}{ Chet X-Ray } & Yes & 13 & 3.8 \\
\hline & No & 327 & 96.2 \\
\hline \multirow{2}{*}{ TB suggestive on Chet X-Ray } & Yes & 5 & 38.5 \\
\hline & No & 8 & 61.5 \\
\hline
\end{tabular}

their current symptom and none of the participants had previous TB treatment history. From 13 (3.8\%) children that were investigated with chest X-ray for their current symptom, 5 (38.7 \%) were found to be suggestive of TB and started on treatment for TB. Of 117 HIV test offered, 98 (83.8\%) were tested but none were reactive for HIV (Table 2).

\subsection{ZN and Xpert MTB/RIF Results}

From 340 children, 1020 samples collected (282 gastric aspirate and 738 sputum samples) were collected. Of these, 38 (3.7\%) were positive by Xpert (10 (3.5\%) from gastric aspirate and $28(3.8 \%)$ from sputum sample. Similarly, 8 (1.2\%) of sputum samples were positive by ZN but none from gastric aspirate. Of 244 children who submitted sputum samples, 3 (1.2\%) were bacteriologically positive compared to 12 (4.9\%) by Xpert (Table 3 ).

Of 96 children who submitted gastric aspirate samples, none were positive by ZN while 5 (5.2\%) were positive by Xpert (Table 4 and Figure 2). 
Table 3. Results of single and pooled sputum samples using ZN and Xpert MTB/RIF.

\begin{tabular}{cccccccccc}
\hline & \multicolumn{3}{c}{ ZN } & \multicolumn{3}{c}{ Xpert MTB/RIF } & ZN: Xpert MTB/RIF** \\
\hline & Negative & Positive & Total & + Pooled N (\%) & MTB- & MTB+ & Total & + +Pooled N (\%) & $\%(\mathrm{n} / \mathrm{N})$ \\
\hline $\mathbf{1}^{\text {st }}$ & 244 & 2 & 246 & $1(33 \%)$ & 238 & 8 & 246 & $3(38 \%)$ & $25 \%(2 / 8)$ \\
$2^{\text {nd }}$ & 243 & 3 & 246 & $0(0 \%)$ & 237 & 9 & 246 & $2(22 \%)$ & $33 \%(3 / 9)$ \\
Pooled $^{*}$ & 243 & 3 & 246 & - & 235 & 11 & 246 & - & $27 \%(3 / 11)$ \\
\hline
\end{tabular}

${ }^{\star}$ Percent of bacteriologically confirmed cases by $\mathrm{ZN}$ is $1.2 \%$, Xpert is $4.5 \%$ with an increase of $3.3 \%$, increase of $0.4 \%$ for $\mathrm{ZN}$ and $1.2 \%$ by $\mathrm{Xpert}$ compared to first sputum sample. ${ }^{*}$ Percentage of smear positive cases detected compared to Xpert MTB/RIF testing.

Table 4. Results of single and pooled gastric aspirate samples using ZN and Xpert MTB/RIF.

\begin{tabular}{cccccccccc}
\hline & \multicolumn{4}{c}{ ZN } & \multicolumn{3}{c}{ Xpert MTB/RIF } & ZN: Xpert MTB/RIF \\
\hline & Negative & Positive & Total & + Pooled N (\%) & MTB- & MTB+ & Total & + Pooled N $(\%)$ & $\%(\mathrm{n} / \mathrm{N})$ \\
\hline $\mathbf{1}^{\text {st }}$ & 94 & 0 & 94 & $0(0 \%)$ & 91 & 3 & 94 & $2(67 \%)$ & $0 \%(0 / 3)$ \\
$2^{\text {nd }}$ & 94 & 0 & 94 & $0(0 \%)$ & 92 & 2 & 94 & $3(60 \%)$ & $0 \%(0 / 2)$ \\
Pooled $^{*}$ & 94 & 0 & 94 & - & 89 & 5 & 94 & - & $0 \%(0 / 5)$ \\
\hline
\end{tabular}

*Percent of bacteriologically confirmed cases by ZN is $0 \%$, Xpert results in increase of 5.3\% (5/94), increase of 0\% for ZN and 2.1\% (2/94) by Xpert compared to first gastric aspirate.

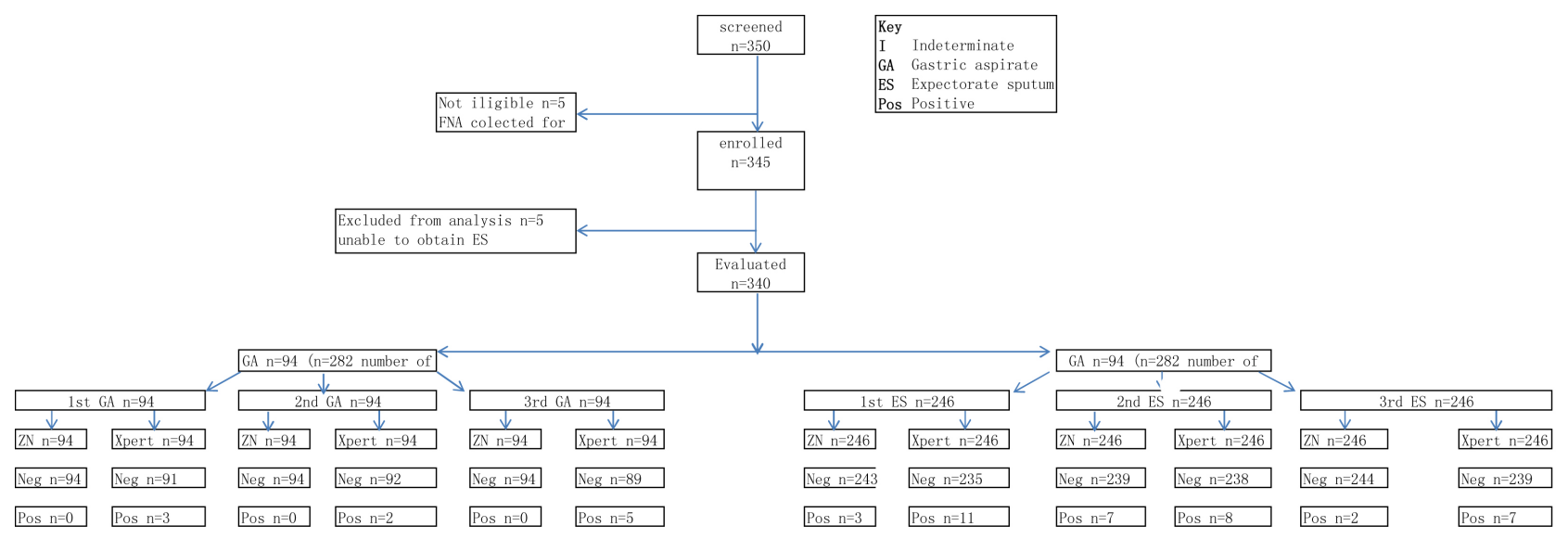

Figure 2. Flow chart showing children diagnosed with bacteriological positive TB.

Pooled sample testing for both sputum and gastric expectorat 3 and 16 bacteriologically confirmed cases were obtained by $\mathrm{ZN}$ and Xpert respectively (Table 5).

Of the 340 children enrolled, 247 sputum samples and 94 gastric aspirates were obtained. $44 \%$ of the children were between 5 - 9 years. No smear positive case was identified in the age group of 0 - 9 years. Xpert tested detected bacteriologically confirmed cases in all age groups, more in pooled samples and gastric aspirates as shown in Table 6.

\section{Discussion}

We report increased yield of bacteriologically confirmed TB cases in pooled samples by Xpert MTB/RIF testing. The overall incremental yield of pooling 
Table 5. Summary results of single and pooled samples using ZN and Gene Xpert.

\begin{tabular}{cccccccccc}
\hline & \multicolumn{3}{c}{ ZN } & \multicolumn{4}{c}{ Xpert MTB/RIF } & \multicolumn{2}{c}{ ZN: Xpert MTB/RIF } \\
\hline & Negative & Positive & Total & +Pooled N (\%) & MTB- & MTB+ & Total & + +Pooled N (\%) & $\%(\mathrm{n} / \mathrm{N})$ \\
\hline $\mathbf{1}^{\text {st }}$ & 338 & 2 & 340 & $1(33 \%)$ & 329 & 11 & 340 & $5(46 \%)$ & $18 \%(2 / 11)$ \\
$\mathbf{2}^{\text {nd }}$ & 337 & 3 & 340 & $0(0 \%)$ & 329 & 11 & 340 & $5(46 \%)$ & $27 \%(3 / 11)$ \\
Pooled $^{*}$ & 337 & 3 & 340 & - & 314 & $16^{* *}$ & 340 & - & $19 \%(3 / 16)$ \\
\hline
\end{tabular}

${ }^{\star}$ Percent of bacteriologically confirmed cases by ZN is $0.9 \%$ (3/340), Xpert is $4.7 \%(16 / 340)$ with an increase of $3.8 \%$, increase of $0.3 \%$ for $\mathrm{ZN}$ and $1.5 \%$ by Xpert of pooled samples compared to first samples. ${ }^{\star \star}$ There was one Xpert MTB/RIF positive sample in the 1st and 2nd samples which ended up negative in pooled test. The overall MTB+ was 17 which is $5 \%$ making an increase of $4.1 \%$.

Table 6. Summary of the type of samples, type of test and results by age category of the study participants.

\begin{tabular}{|c|c|c|c|c|c|c|c|c|c|c|c|c|c|}
\hline \multirow[t]{2}{*}{ age category } & \multirow[t]{2}{*}{ Participants } & \multicolumn{2}{|c|}{$1^{\text {st }}$ smear } & \multicolumn{2}{|c|}{$2^{\text {nd }}$ smear } & \multicolumn{2}{|c|}{ Pooled smear } & \multicolumn{2}{|c|}{$1^{\text {st }}$ Xpert } & \multicolumn{2}{|c|}{$2^{\text {nd }}$ Xpert } & \multicolumn{2}{|c|}{ Pooled Xpert } \\
\hline & & NEG & POS & NEG & POS & NEG & POS & MTB- & MTB+ & MTB- & MTB+ & MTB- & MTB+ \\
\hline $0-4$ & 76 & 76 & 0 & 76 & 0 & 76 & 0 & 73 & 3 & 74 & 2 & 71 & 5 \\
\hline $5-9$ & 149 & 149 & 0 & 149 & 0 & 149 & 0 & 147 & 2 & 146 & 3 & 144 & 5 \\
\hline $10-14$ & 115 & 113 & 2 & 112 & 3 & 112 & 3 & 109 & 6 & 109 & 6 & 109 & 6 \\
\hline Total & 340 & 338 & 2 & 337 & 3 & 337 & 3 & 329 & 11 & 329 & 11 & 324 & 16 \\
\hline
\end{tabular}

samples was $0.9 \%$ for smear microscopy and $4.7 \%$ for Xpert. Compared to first samples, smear microscopy and Xpert increased the yield by $0.3 \%$ and $1.5 \%$ respectively.

In our study bacteriologically positive TB case were not identified in gastric aspirates compared to $1 \%-13 \%$ smear positive TB identified in other studies [17] [18] [19] [20]. This could be due to low bacilli load and low sensitivity of AFB smear [21] [22].

The yield of bacteriological conformation was high in sputum samples compared to gastric aspirates [23]. In this study the proportion of sputum smear positive in the first and second samples $(0.8 \%$ and $1.2 \%$ respectively) were low in agreement with findings in other similar studies ranged from $4 \%-10.7 \%$ to $0.32 \%-5 \%$ for the first and second sputum samples respectively [17] [24] [25]. Sputum microscopy detected cases in the age group of 9 - 14 years. This could be due to the capacity to expectorate and more bacillary load. Likewise, Xpert detected cases in children less than five years. This could be due to increased sensitivity.

Same day smear microscopy for diagnosing tuberculosis in the case of suspected tuberculosis seems not a promising step towards improving the quality of smear examination [26]. In our study Xpert MTB/RIF detects more positive than smear microscopy similar to other studies [27] [28]. Because of the highly sensitivity Xpert MTB/RIF [28] [29] [30], overcame the dilution effect of pooling samples. The process of collecting samples in one hour difference (front loading approach) could break a major impediment to treatment of TB is a diagnostic process that requires multiple visits [31]. This data indicates that the strategy of 
collecting specimen in one hour interval and pooling samples which were tested together has reduced diagnostic visits and could improve compliance in submitting samples and collecting result. It can also decrease the laboratory workload and hospitalization and hence improve the quality of work in settings with high TB burden like Ethiopia.

Confirmatory diagnosis of children tuberculosis is a medical challenge and the diagnosis of childhood tuberculosis (TB) is complex [32]. Therefore, alternative approaches that reduce the number of visits by patients and improve efficiency of laboratory professionals could contribute to improving confirmation of bacteriologically confirmed cases with short turnaround time.

Limitations of the study were that the number of participants for sputum examination was older than those tested by Xpert. Therefore, it was not possible to compare the overall sensitivity of ZN to Xpert test.

\section{Conclusion}

The use of Xpert for samples from children increases yield in pooled samples. The same-day approach and the use of a single cartridge for testing multiple samples of a single child using Xpert MTB/RIF reduce the number of visits required for diagnosis, have the potential to save resources for the health system and the patient, and ultimately improve case detection in poorer countries. GeneXpert MTB/RIF could be an alternative for testing pooled sample to increase the detection and confirmation of children with TB.

\section{Acknowledgements}

The authors are sincerely grateful to all health workers for their active participation in collecting the data.

\section{Authors' Contributions}

DG and LE conceived the study. MY, MD and MA wrote the manuscript. DG, LE and KT revised it critically for intellectual content and gave final approval of the version to be published. All authors read and approved the final manuscript.

\section{Funding}

The study was funded by the Grand Challenges Canada Grant ID S4 0252-01.

\section{Conflicts of Interest}

The authors declare that they have no competing interests for this work.

\section{References}

[1] WHO (2017) Global Tuberculosis Report. WHO, Geneva.

[2] WHO (2016) Global Tuberculosis Report. WHO, Geneva.

[3] Nelson, L.J. and Wells, C.D. (2004) Global Epidemiology of Childhood Tuberculosis. The International Journal of Tuberculosis and Lung Disease: The Official Jour- 
nal of the International Union against Tuberculosis and Lung Disease, 8, 636-647.

[4] WHO (2006) Guidlines for National Tuberculosis Programmes on the Management of Tuberculosis Programmes on the Management of Tuberculosis in Children. WHO, Geneva.

[5] WHO (2015) Global Tuberculosis Report. 20th Edition, WHO, Geneva.

[6] Enarson, P.M., Enarson, D.A. and Gie, R. (2005) Management of Tuberculosis in Children in Low-Income Countries. The International Journal of Tuberculosis and Lung Disease: The Official Journal of the International Union against Tuberculosis and Lung Disease, 9, 1299-1304.

[7] Graham, S.M., Gie, R.P., Schaaf, H.S., Coulter, J.B., Espinal, M.A. and Beyers, N. (2004) Childhood Tuberculosis: Clinical Research Needs. The International Journal of Tuberculosis and Lung Disease: The Official Journal of the International Union against Tuberculosis and Lung Disease, 8, 648-657.

[8] Dangisso, M.H., Datiko, D.G. and Lindtjorn, B. (2015) Low Case Notification Rates of Childhood Tuberculosis in Southern Ethiopia. BMC Pediatrics, 15, 142.

https://doi.org/10.1186/s12887-015-0461-1

[9] WHO (2002) An Expanded DOTS Framework for Effective Tuberculosis Control. WHO, Geneva.

[10] CDC (2014) Reported Tuberculosis in the United States, 2013. U.S. Department of Health and Human Services, CDC, Atlanta, GA.

[11] Marais, B.J. (2004) Childhood Turberculosis: Reflections from the Front Line. Pediatric Annals, 33, 695-698. https://doi.org/10.3928/0090-4481-20041001-13

[12] Abdurrahman, S.T., Mbanaso, O., Lawson, L., Oladimeji, O., Blakiston, M., Obasanya, J., Dacombe, R., Adams, E.R., Emenyonu, N., Sahu, S., et al. (2015) Testing Pooled Sputum with Xpert MTB/RIF for Diagnosis of Pulmonary Tuberculosis to Increase Affordability in Low-Income Countries. Journal of Clinical Microbiology, 53, 2502-2508. https://doi.org/10.1128/JCM.00864-15

[13] Tom, S.K., Welch, H., Kilalang, C., Tefuarani, N., Vince, J., Lavu, E., Johnson, K., Magaye, R. and Duke, T. (2017) Evaluation of Xpert MTB/RIF Assay in Children with Presumed Pulmonary Tuberculosis in Papua New Guinea. Paediatrics and International Child Health, 38, 97-105.

[14] Soham, G., Vishnu, P.S., Indira, B. and Muralidharan, S. (2010) Diagnostic Efficacy of Ziehl-Neelsen Method Against Fluorescent Microscopy in Detection of acid Fast Bacilli. Asian Pacific Journal of Tropical Medicine, 3, 328-329. https://doi.org/10.1016/S1995-7645(10)60079-X

[15] Iram, S., Zeenat, A., Hussain, S., Wasim Yusuf, N. and Aslam, M. (2015) Rapid Diagnosis of Tuberculosis Using Xpert MTB/RIF Assay-Report from a Developing Country. Pakistan Journal of Medical Sciences, 31, 105-110.

[16] Tenover, F. (2009) Xpert ${ }^{\oplus}$ MTB/RIF Two-Hour Detection of MTB and Resistance to Rifampicin.

[17] Al-Aghbari, N., Al-Sonboli, N., Yassin, M.A., Coulter, J.B., Atef, Z., Al-Eryani, A. and Cuevas, L.E. (2009) Multiple Sampling in One Day to Optimize Smear Microscopy in Children with Tuberculosis in Yemen. PLoS ONE, 4, e5140. https://doi.org/10.1371/journal.pone.0005140

[18] Cruz, A.T., Revell, P.A. and Starke, J.R. (2013) Gastric Aspirate Yield for Children with Suspected Pulmonary Tuberculosis. Journal of the Pediatric Infectious Diseases Society, 2, 171-174. https://doi.org/10.1093/jpids/pis089

[19] Parashar, D., Kabra, S.K., Lodha, R., Singh, V., Mukherjee, A., Arya, T., Grewal, 
H.M. and Singh, S. (2013) Does Neutralization of Gastric Aspirates from Children with Suspected Intrathoracic Tuberculosis Affect Mycobacterial Yields on MGIT Culture? Journal of Clinical Microbiology, 51, 1753-1756. https://doi.org/10.1128/JCM.00202-13

[20] Gomez Pastrana Duran, D., Torronteras Santiago, R., Caro Mateo, P., Lopez Barrio, A.M., Macias Mardones, P., Andres Martin, A., Pineda Mantecon, M. and Navarro Gonzalez, J. (2000) Effectiveness of Smears and Cultures in Gastric Aspirate Samples in the Diagnosis of Tuberculosis. Anales Espanoles de Pediatria, 53, 405-411. https://doi.org/10.1016/S1695-4033(00)78620-6

[21] Kalu, E.I., Ojide, C.K. and Nwadike, V.U. (2014) Validity of Acid-Fast Smear of Gastric Aspirates for the Diagnosis of Childhood Pulmonary Tuberculosis among Human Immunodeficiency Virus-Infected Children. International Journal of Tropical Disease \& Health, 4, 111-122. https://doi.org/10.9734/IJTDH/2014/5966

[22] Elhassan, M.M., Elmekki, M.A., Osman, A.L. and Hamid, M.E. (2015) Challenges in Diagnosing Tuberculosis in Children: A Comparative Study from Sudan. International Journal of Infectious Diseases, 43, 25-29.

[23] Berggren Palme, I., Gudetta, B., Bruchfeld, J., Eriksson, M. and Giesecke, J. (2004) Detection of Mycobacterium tuberculosis in Gastric Aspirate and Sputum Collected from Ethiopian HIV-Positive and HIV-Negative Children in a Mixed in- and Out-Patient Setting. Acta Paediatrica, 93, 311-315.

[24] Seni, J., Kidenya, B.R., Obassy, E., Mirambo, M., Burushi, V., Mazigo, H.D., Kapesa, A., Majigo, M. and Mshana, S.E. (2012) Low Sputum Smear Positive Tuberculosis among Pulmonary Tuberculosis Suspects in a Tertiary Hospital in Mwanza, Tanzania. Tanzania Journal of Health Research, 14, 115-120. https://doi.org/10.4314/thrb.v14i2.4

[25] Islam, M.R., Khatun, R., Uddin, M.K., Khan, M.S., Rahman, M.T., Ahmed, T. and Banu, S. (2013) Yield of Two Consecutive Sputum Specimens for the Effective Diagnosis of Pulmonary Tuberculosis. PLOS ONE, 8, e67678. https://doi.org/10.1371/journal.pone.0067678

[26] Myneedu, V.P., Verma, A.K., Sharma, P.P. and Behera, D. (2011) A Pilot Study of Same Day Sputum Smear Examination, Its Feasibility and Usefulness in Diagnosis of Pulmonary TB. The Indian Journal of Tuberculosis, 58, 160-167.

[27] Geleta, D.A., Megerssa, Y.C., Gudeta, A.N., Akalu, G.T., Debele, M.T. and Tulu, K.D. (2015) Xpert MTB/RIF Assay for Diagnosis of Pulmonary Tuberculosis in Sputum Specimens in Remote Health Care Facility. BMC Microbiology, 15, 220. https://doi.org/10.1186/s12866-015-0566-6

[28] Ciftci, I.H., Aslan, M.H. and Asik, G. (2011) Evaluation of Xpert MTB/RIF Results for the Detection of Mycobacterium tuberculosis in Clinical Samples. Mikrobiyoloji Bulteni, 45, 43-47.

[29] Singh, S., Singh, A., Prajapati, S., Kabra, S.K., Lodha, R., Mukherjee, A., Singh, V., Hesseling, A.C. and Grewal, H.M. (2015) Xpert MTB/RIF Assay Can Be Used on Archived Gastric Aspirate and Induced Sputum Samples for Sensitive Diagnosis of Paediatric Tuberculosis. BMC Microbiology, 15, 191. https://doi.org/10.1186/s12866-015-0528-Z

[30] Bojang, A.L., Mendy, F.S., Tientcheu, L.D., Out, J., Antonio, M., Kampmann, B., Agbla, S. and Sutherland, J.S. (2015) Comparison of TB-LAMP, GeneXpert MTB/RIF and Culture for Diagnosis of Pulmonary Tuberculosis in the Gambia. The Journal of Infection, 72, 332-337.

[31] de Cuevas, R.M., Lawson, L., Al-Sonboli, N., Al-Aghbari, N., Arbide, I., Sherchand, 
J.B., Nnamdi, E.E., Aseffa, A., Yassin, M.A., Abdurrahman, S.T., et al. (2016) Patients Direct Costs to Undergo TB Diagnosis. Infectious Diseases of Poverty, 5, 24. https://doi.org/10.1186/s40249-016-0117-x

[32] Cuevas, L.E., Petrucci, R. and Swaminathan, S. (2012) Tuberculosis Diagnostics for Children in High-Burden Countries: What Is Available and What Is Needed. Paediatrics and International Child Health, 32, S30-S37.

https://doi.org/10.1179/2046904712Z.00000000076 